Research Article

\title{
Gust Perturbation Alleviation Control of Small Unmanned Aerial Vehicle Based on Pressure Sensor
}

\author{
Zijun Ren (D), Wenxing Fu, and Jie Yan \\ School of Astronautics, Northwestern Polytechnical University, Xi'an 710072, China \\ Correspondence should be addressed to Zijun Ren; zijunr@gmail.com
}

Received 13 September 2017; Revised 19 April 2018; Accepted 23 April 2018; Published 2 July 2018

Academic Editor: Mahmut Reyhanoglu

Copyright (c) 2018 Zijun Ren et al. This is an open access article distributed under the Creative Commons Attribution License, which permits unrestricted use, distribution, and reproduction in any medium, provided the original work is properly cited.

\begin{abstract}
A gust perturbation alleviation control method based on a real-time pressure sensor is proposed. Pressure measurement provides phase-advance information on external disturbance, while the conventional inertial measurement cannot. Two pairs of pressure sensors embedded on the main wing surfaces are employed to estimate the disturbance of gust-induced rolling moment. The estimated rolling moment is incorporated into a traditional flight controller as an additional feedforward channel. The simulation results show that the additional information on flow field is helpful and that the composite controller's architecture is more effective for alleviating gust perturbation than the conventional ones.
\end{abstract}

\section{Introduction}

Various varieties of unmanned aerial vehicles (UAVs) are increasingly common in daily life, especially the small ones, such as selfie quadrotor, delivery drone, power line inspection flying robot, and forest fire alarm unmanned flight vehicle. A small unmanned aerial vehicle (SUAV) has a wingspan of about 0.6 to $5 \mathrm{~m}$ and a gross mass of about 0.5 to $20 \mathrm{~kg}$ [1]. The application scenarios of SUAV include a variety of regions like indoor, urban, forest and mountainous regions, and seaside. A SUAV usually flies near the ground, where buildings and trees may reach its altitude. Its low speed demands that it may fly in the flight regime of the low Reynolds number. Its flight safety is threatened by the complex near-ground environment and unpredictable air flow conditions. Its frequent crash incidents indicate that the traditional flight control strategies designed for a conventional large aircraft are not suitable for SUAV's flight control due to its low-speed, lightweight, coarse sensor, and low-cost actuator [2].

Researchers and engineers propose hundreds of methods to improve an SUAV's flight performance [2-4]. Some researchers get their inspirations from the nature, such as flying creatures like birds and insects which are capable of high-performance flight. Birds and insects have plentiful flow receptors all round their bodies and wings; that is why they are endowed with the ability to sense the information on flow near their bodies. Mohamed et al. classified sensors according to gust perturbation processes [5], while a phase-advance sensor like flow sensor and strain sensor can detect disturbance before the inertial response of the airframe. The reactive nature of the widely used traditional inertial sensor, nevertheless, causes time lag in alleviating gust disturbance. An effective method is to improve the flight performance of the SUAV in turbulence by reducing its response delay. Shen et al. utilized two lines of onboard airflow sensors to sense real-time pressure and shear stress. And simulation results show that the adaptive robust control system proposed in [6] is able to maintain stability, even if half of its sensors malfunction. Pressure sensors throughout the wing surfaces can provide accurate moment estimates under hover and forward-flight conditions and enhance the real-time pitch and yaw control [7]. Araujo-Estrada et al. tested two SUAV platforms embedded with strain sensors and pressure sensors. Their advantages include stall detection, gust mitigation, and aerodata measurement are revealed [8]. A load sensor on the aerodynamic surfaces can remarkably improve robustness and flight performance [9]. Experiments conducted by Castano et al. verified the rapidity of structural feedback; the controller of the experimental SUAV installed 


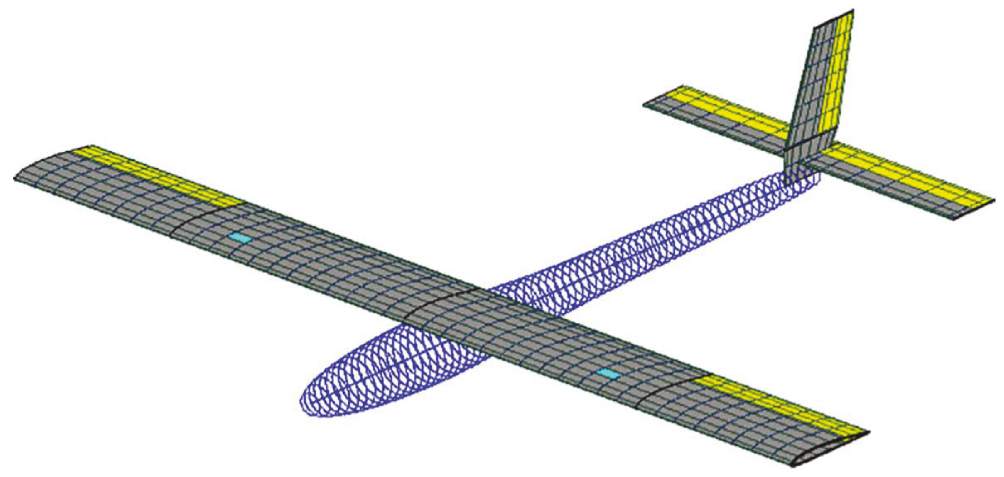

FIGURE 1: The vortex lattice model of an experimental SUAV with pressure sensors marked by blue spots on the main wing surfaces.

with strain gauge feedback shows that it is more effective for suppressing vertical gust disturbance than the conventional rigid body controller [10]. A small quadrotor was used to exploit the ability to estimate force and torque via distributed acceleration and strain sensing. It is proved that the feedback from the estimation can alleviate rolling perturbation and heave perturbation [11]. The LiDAR technology, though far from applicable to SUAV, is proved to be promising in turbulence attenuation $[12,13]$.

The goal of the current research is to investigate the potentials of a pressure sensor for improving the flight stability of a SUAV in a gusty environment. First, the SUAV is presented; the locations of the pressure sensors are designed elaborately. Then, the gust's rolling moment estimation algorithm is discussed; the composite feedforward-feedback controller is designed. Finally, the linear and nonlinear control simulation results demonstrate the feasibility of the gust perturbation alleviation control method.

\section{The SUAV with Pressure Sensors}

The experiments on a conventional lightweight wing-tail configuration of a model plane as an example of a SUAV were conducted, using the designed trim velocity of $20 \mathrm{~m} / \mathrm{s}$, the wingspan of $1.27 \mathrm{~m}$, and the gross mass of $2 \mathrm{~kg}$, as shown in Figure 1. The trim angle of attack $\alpha$ is about 1.5 degree. The actuator is modeled as a second-order system

$$
G_{\mathrm{a}}=\frac{1}{0.01^{2} s^{2}+2 \cdot 0.7 \cdot 0.01 \cdot s+1} .
$$

The position of a pressure sensor must be selected elaborately to provide aircraft surface pressure distribution for estimating aerodynamic forces and moments. It is better to position the pressure sensor at the position that is sensitive to pressure change. Vogel and Kelkar positioned the upper and lower surface ports of a FADS system near the leading edge of its airfoil chordwise. They placed the surface ports at two stations spanwise (about $1 / 4$ and $1 / 2$ semispanwise positions from the center) [14]. Their work shows that the local lift magnitudes near the leading edge of the airfoil vary rapidly with the airfoil's section lift coefficient. Marino et al. placed four modules in the forepart of the wing chordwise and two locations near the center spanwise [15]. Ten pressure taps are installed in each module. The wind tunnel experimental results prove the correlation between measured unsteady pressure and total lift force of the wing. Shen et al. choose to set 10 pressure sensors along the mean aerodynamic chord [6]. The experimental results implemented by Mohamed et al. reveal that the leading edge is suitable for placing pressure taps and that it is better to use the location at the maximum lift section along the wingspan to estimate the lift and rolling moment of the SUAV [16].

In this study, two pairs of pressure sensors were embedded symmetrically in the upper and lower wing surfaces, as shown in Figure 1. The two pressure sensors in each pair were located at the same chord locations of the upper and lower wing surfaces. In the experiments on SUAVs, as few pressure sensors as possible were chosen to reduce the complexity of wire connection. To enhance the performance of the gust perturbation alleviation control method that uses few sensors, based on the previous work, the locations of the two pairs of sensors were selected spanwise at about $1 / 2$ semiwingspan and chordwise at about $25 \%$ chord near the leading edge. Several analyses of the 2D flow fields of the SUAV airfoil (AG35) were carried out in XFOIL. Figure 2 gives two scenarios of properly placing pressure sensors on the airfoil chord. Differences in pressure on the upper and lower wing surfaces as measured by the sensors can be used to replace the mean difference in pressure on the whole wing surface approximately. Lift and rolling moments can be estimated with the measured difference in pressure. Although there is much room for improvement to reduce estimation errors, the principles revealed are helpful.

\section{The Gust's Rolling Moment Estimation}

The aerodynamic force and moment of a moving object in the atmosphere are generated by the interaction between the object's surface and the air. All the aerodynamic force and moment can be obtained with surface pressure distribution except drag. Because there is a limited number of sensors, it is difficult to obtain accurate surface pressure distribution. The blue dashed box in Figure 2 represents the sensor measurements, and the black solid line represents 


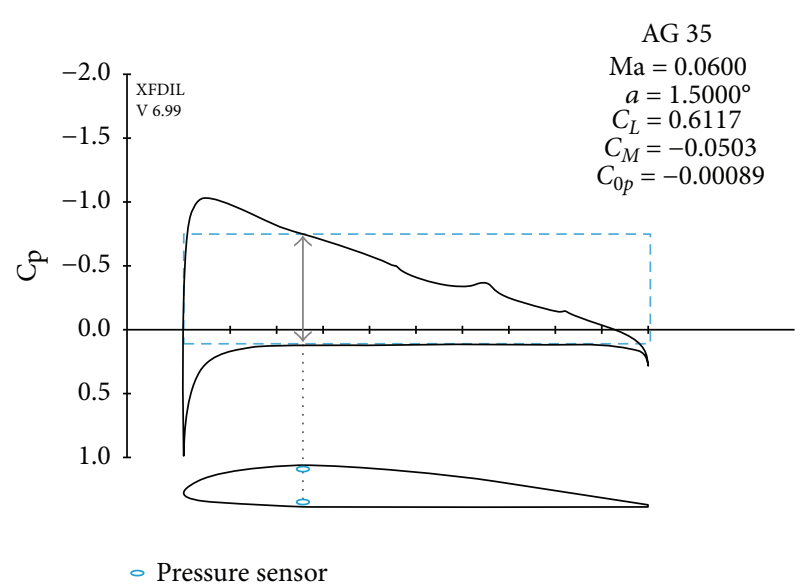

(a) $\alpha=1.5^{\circ}$

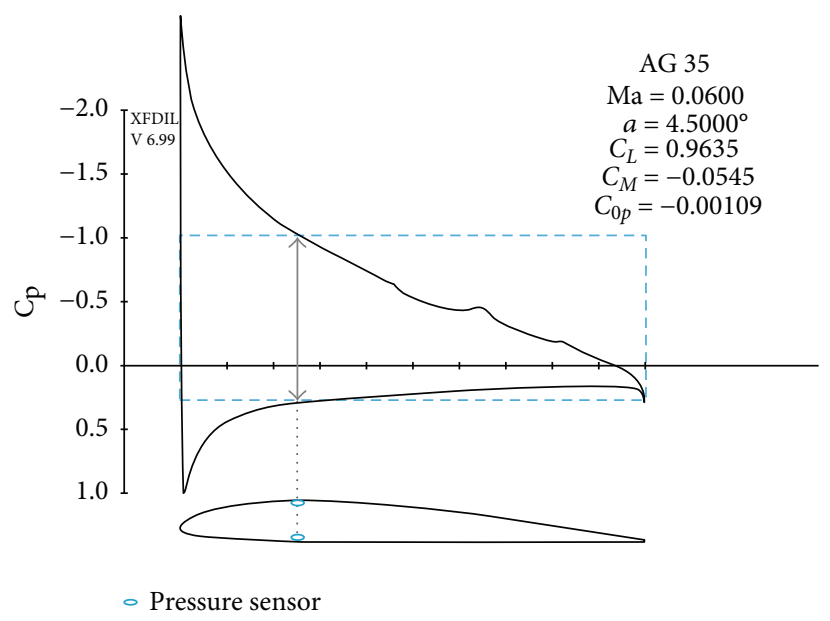

(b) $\alpha=4.5^{\circ}$

FIGURE 2: Differences in pressure on the upper and lower main wing surfaces, with blue circles representing pressure sensors.

the accurate pressure distribution. Nevertheless, the two sensor measurements of the upper and lower wing surfaces can produce relatively reliable results against accurate pressure distribution.

It is firmly believed that an SUAV is most susceptible in rolling motion in a gusty environment [17]. So, in this work, the main research object is to estimate and attenuate the gust-induced rolling moment.

The SUAV under experiments has a straight main wing with the AG35 airfoil section. The wing angle of incidence is 0 degree; the normal force vector $\mathbf{N}_{i}$ of the wingspan per unit is

$$
\mathbf{N}_{i}=\int_{x_{\mathrm{LE}}}^{x_{\mathrm{TE}}}\left(p_{\text {low }}-p_{\text {up }}\right) d x
$$

where the normal force vector $\mathbf{N}_{i}$ is parallel to the $z$ body axis and positive upward; the subscripts LE and TE stand for the leading edge and trailing edge; $p_{\text {low }}$ and $p_{\text {up }}$ denote the pressure distributions of the lower and upper wing surfaces, respectively; $x$ represents the longitudinal body axis.

The total rolling moment of a SUAV consists mainly of the rolling moments on the main wing surface, the tail, and the fuselage and can be expressed as follows:

$$
L_{\text {total }}=L_{\text {wing }}+L_{\text {tail }}+L_{\text {fuselage }} \text {, }
$$

where $L_{\text {wing }}$ is the total rolling moment of the main wing. Hence, it can be resolved through integrating the normal force vector $\mathbf{N}_{i}$ at different spanwise sections across the wingspan and is expressed as follows:

$$
\begin{aligned}
& L_{\text {total }} \approx L_{\text {wing }}, \\
& L_{\text {wing }}=-\int_{\text {span }}\left(N_{i} \cdot y\right) d y,
\end{aligned}
$$

where $y$ is the $y$ body coordinate. $L_{\text {wing }}$ can be linearly decomposed into the control torque $L_{\text {ail }}$ and the gust-induced rolling moment $L_{\text {gust }}$. Therefore, the latter can be regarded as an additional perturbation item to the SUAV's rolling moment and expressed as follows:

$$
L_{\text {total }} \approx L_{\text {ail }}+L_{\text {gust }} \text {. }
$$

Since the aileron deflection may change the distribution of pressure on a section of the wing surface, the only pair of sensors is deliberately embedded at the 1/2 semiwingspan location away from the aileron, as shown in Figure 1. To formulate the gust-induced rolling moment $L_{\text {gust }}$, we substitute the measurement by the pair of sensors with low-pass filters for transient pressure distribution. The $L_{\text {gust }}$ can be estimated by the following expression:

$$
\begin{aligned}
\widehat{L}_{\text {gust }}= & -\underbrace{\int_{\text {left wing }}\left(\hat{p}_{\text {low }}-\widehat{p}_{\text {up }}\right) \cdot y \cdot c d y}_{\text {left sensor pair }} \\
& -\underbrace{\int_{\text {right wing }}\left(\hat{p}_{\text {low }}-\widehat{p}_{\text {up }}\right) \cdot y \cdot c d y}_{\text {right sensor pair }},
\end{aligned}
$$

where $\widehat{p}_{*}$ is the measurement by the pair of sensors with lowpass filters; $c$ is the wing chord. The reformulation of $\widehat{L}_{\text {gust }}$ uses a simple form, as shown below:

$$
\widehat{L}_{\text {gust }}=\frac{c \cdot b^{2}}{8}\left(\Delta \widehat{p}_{1}-\Delta \widehat{p}_{\mathrm{r}}\right),
$$

where $b$ is the wingspan; $\Delta \widehat{p}_{1}$ is the difference of pressure on the left half of the wing, which is measured by the pair of 


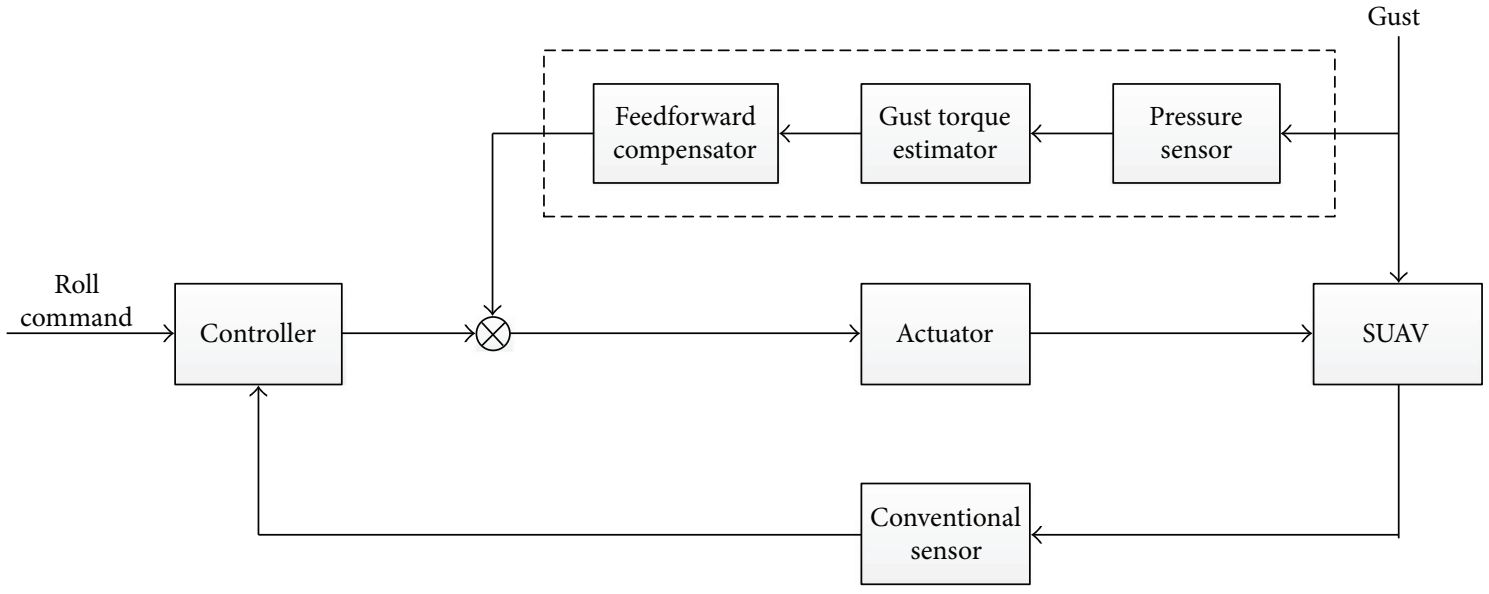

FIGURE 3: The pressure sensor-based gust perturbation alleviation control architecture.

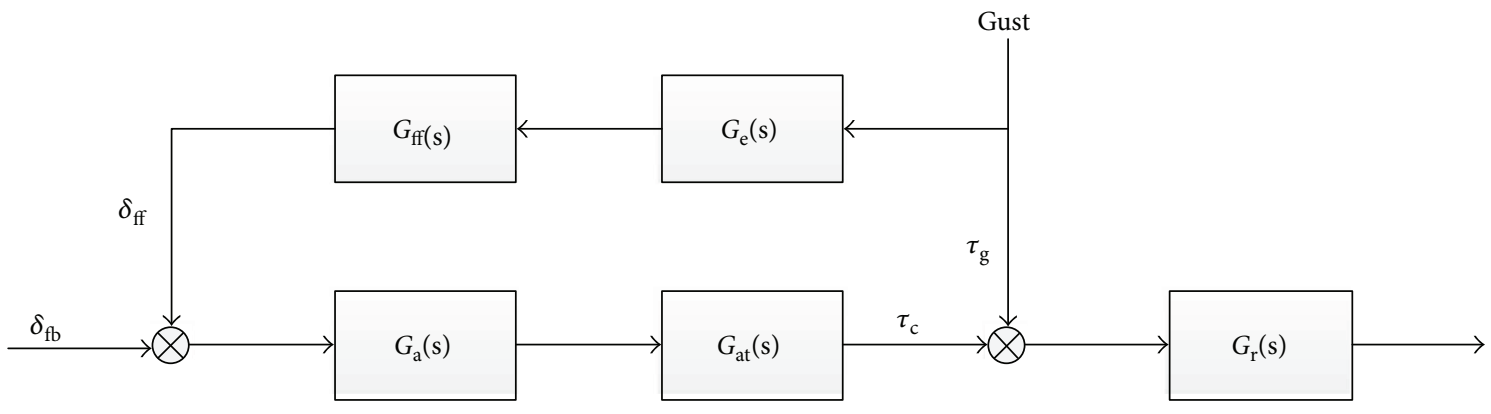

FIGURE 4: The block diagram for feedforward control architecture.

sensors; $\Delta \widehat{p}_{\mathrm{r}}$ is that on the right half of the wing. $\Delta \widehat{p}_{1}$ and $\Delta \widehat{p}_{\mathrm{r}}$ are obtained with the following:

$$
\begin{gathered}
\Delta \widehat{p}_{1}=\widehat{p}_{\text {low }}-\left.\widehat{p}_{\text {up }}\right|_{\text {left pair }}, \\
\Delta \widehat{p}_{\mathrm{r}}=\widehat{p}_{\text {low }}-\left.\widehat{p}_{\text {up }}\right|_{\text {right pair }} .
\end{gathered}
$$

\section{The Feedforward Control Architecture}

As revealed previously, a SUAV is susceptible to rolling motion; therefore, this paper proposes a pressure sensorbased gust perturbation alleviation composite control architecture, as shown in Figure 3, which is made up of the conventional inertial sensor-based feedback channel and the additional pressure-sensing feedforward channel. The latter provides the phase-advance information that an inertial measurement cannot offer.

The feedforward control architecture is shown in Figure 4. In the figure, $G_{\mathrm{a}}(s), G_{\mathrm{at}}(s)$, and $G_{\mathrm{r}}(s)$ denote the transfer functions between aileron command to aileron deflection, aileron deflection to rolling moment, and rolling moment to rolling angular velocity, respectively. $G_{\mathrm{e}}(s)$ represents the gust moment estimator. $G_{\mathrm{ff}}(s)$ is the gust-induced rolling moment feedforward compensator. $\delta_{\mathrm{fb}}$ and $\delta_{\mathrm{ff}}$ are feedback control command and additional feedforward control command, respectively. $\tau_{\mathrm{c}}$ and $\tau_{\mathrm{g}}$ are control moment and gust-induced moment, respectively.

According to the classical control theory, $G_{\mathrm{r}}(s)$ should be independent of gust perturbation if $G_{\mathrm{ff}}(s)$ satisfies the following relationship:

$$
G_{\mathrm{ff}}(s)=-\frac{1}{G_{\mathrm{e}}(s) G_{\mathrm{a}}(s) G_{\mathrm{at}}(s)}
$$

Nevertheless, it seems impossible to implement $G_{\mathrm{ff}}(s)$ in (10), which needs to be simplified for compromise. $G_{\mathrm{a}}(s)$ and $G_{\mathrm{e}}(s)$ are neglected for their fast dynamic responses. $G_{\mathrm{at}}(s)$ is simplified into $\partial \tau / \partial a$ (i.e., aileron control moment per unit of deflection), which depends on the SUAV's aerodynamic coefficient Cla. The feedforward compensator is designed as follows:

$$
G_{\mathrm{ff}}(s)=-\frac{1}{\partial \tau / \partial a}
$$

In order to verify the advantages of the composite feedforward control architecture, the SUAV's attitude control is implemented by three independent multiloop controllers. In addition, a PD altitude-keeping controller is added to the outer loop to maintain level flight. 


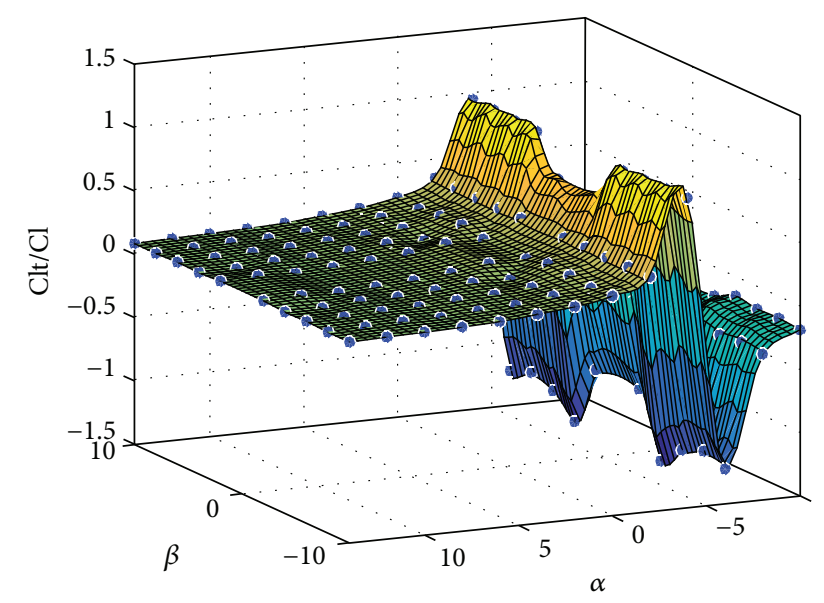

FIGURE 5: The ratio between the rolling moment generated by the tail (Clt) and total rolling moment $(\mathrm{Cl})$.

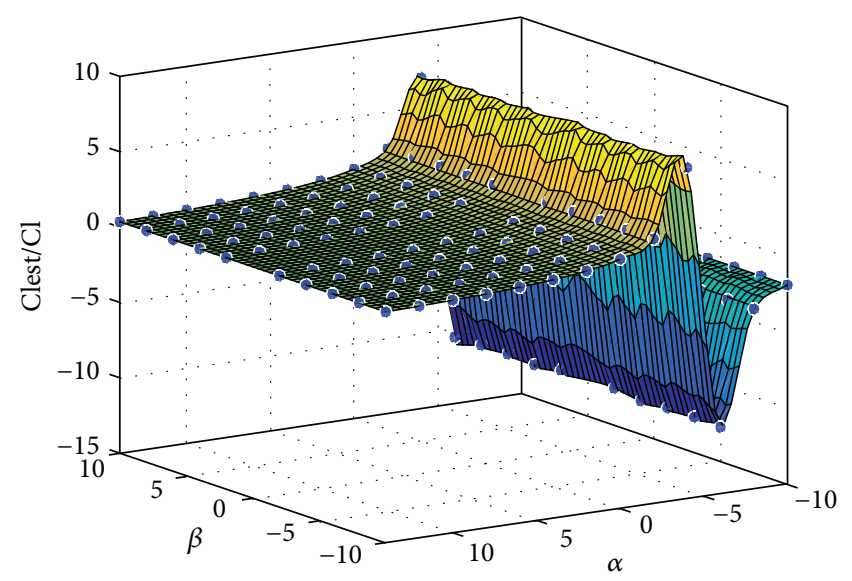

Figure 6: The ratio between estimated rolling moment (Clest) and total rolling moment $(\mathrm{Cl})$.

\section{Implementation and Simulation}

The simulations were carried out with MATLAB ${ }^{\mathrm{TM}}$ and Simulink ${ }^{\mathrm{TM}}$. The SUAV's aerodynamic characterization was accomplished with the AVL [18] program package, which can provide the pressure distribution profile.

Figure 5 shows the ratio between rolling moment generated by the tail $\left(L_{\text {tail }}\right)$ and total rolling moment $\left(L_{\text {total }}\right)$ discussed by (3). It reveals that the rolling moment generated by the tail changed drastically when the angle of attack is around 0 degree. Nevertheless, the sideslip angle has less effect on the tail's rolling moment. When the angle of attack is larger than the critical angle, the proportion of the tail's rolling moment is less than $25 \%$. The greater the angle of attack is, the less the ratio of the rolling moment is. The approximation of (4) is reasonable only when the angle of attack is larger than 0 degree.

Figure 6 depicts the ratio between the estimated rolling moment $\left(\widehat{L}_{\text {gust }}\right)$ and total rolling moment $\left(L_{\text {total }}\right)$. The ratio has a similar variation trend to the tail's rolling moment. The estimation is unauthentic when the angle of attack is less

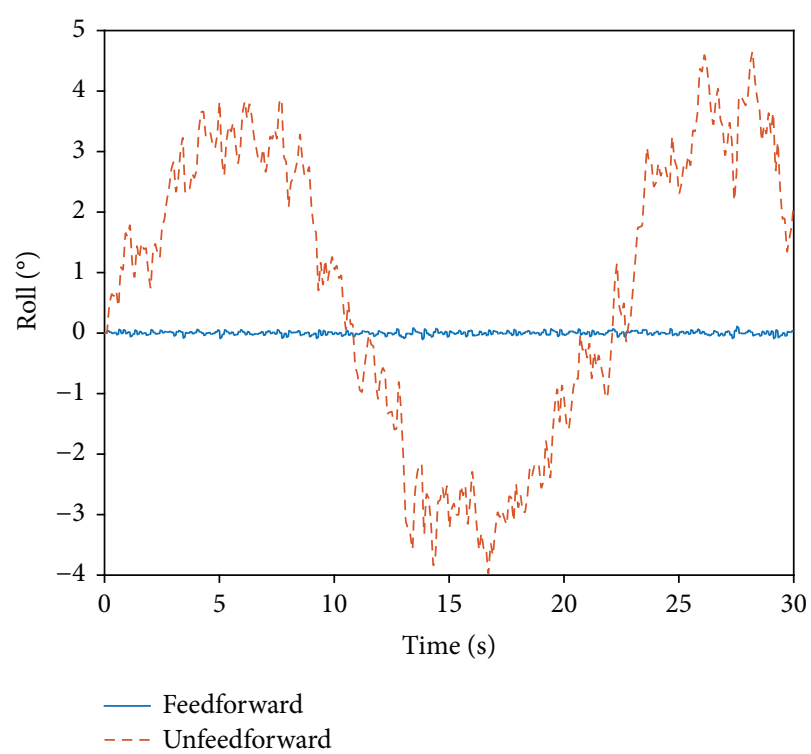

FIGURE 7: The feedforward and unfeedforward control with the linear model.

than -1 degree and substantially undervalued when the angle of attack is larger than 7 degrees, which means $\widehat{L}_{\text {gust }} \leq 0.5 L_{\text {total }}$.

Furthermore, a correction factor $f_{\text {trim }}$ is needed to amend the estimation equation (8). Figure 2(a) shows that the estimated pressure distribution (the blue dashed box) is not equal to the real pressure distribution (the black solid profile) under the trim flight condition. $f_{\text {trim }}$ is introduced to match the estimation and the real value and calculated with the following:

$$
f_{\text {trim }}=\left.\frac{\widehat{L}_{\text {gust }}}{L_{\text {total }}}\right|_{\alpha=1.5^{\circ}} \text {. }
$$

In the above equation, $\alpha$ is chosen as the trim flight attack angle. (8) is amended as follows:

$$
\widehat{L}_{\text {gust }}=f_{\text {trim }} \cdot \frac{c \cdot b^{2}}{8}\left(\Delta \widehat{p}_{1}-\Delta \widehat{p}_{\mathrm{r}}\right) .
$$

To demonstrate the capability of the pressure sensorbased gust perturbation alleviation control method to eliminate gust perturbation, two simulation instances are presented. The linear control simulation instance gives the simulation results on the linear model of the rolling motion controller; the 6-DOF control simulation instance gives the simulation results on the nonlinear control model of the SUAV.

5.1. The Linear Control Simulation Instance. The linearized rolling motion model is a two-order linear model. The rolling motion controller has two feedback control loops: the rolling angular velocities $p$ and the roll angle $\phi$. The controller's gain parameters are designed for the following trim flight conditions: the velocity is $20 \mathrm{~m} / \mathrm{s}$; the altitude is $100 \mathrm{~m}$. The feedforward command is added to the aileron actuator. 


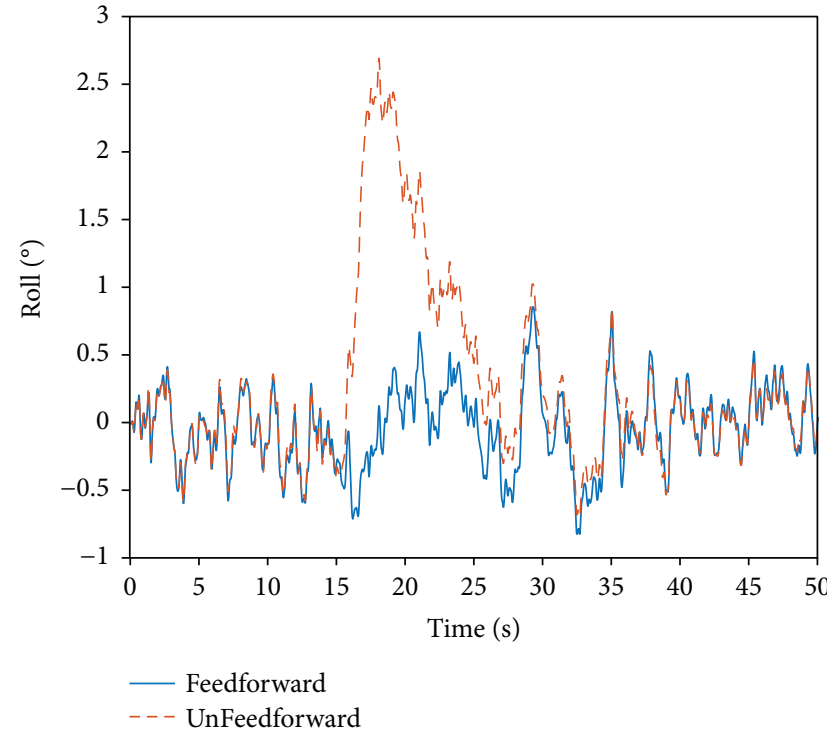

(a) The $+10 \mathrm{~m} / \mathrm{s}$ lateral gust

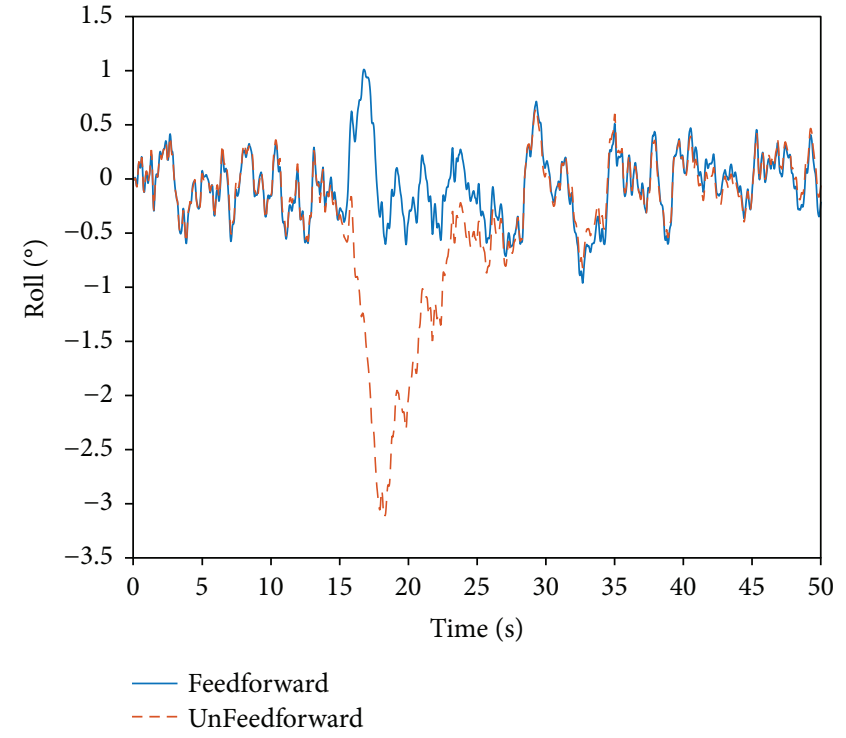

(b) The $-10 \mathrm{~m} / \mathrm{s}$ lateral gust

FIGURE 8: The feedforward and unfeedforward rolling motion control with the nonlinear 6-DOF model of the SUAV.

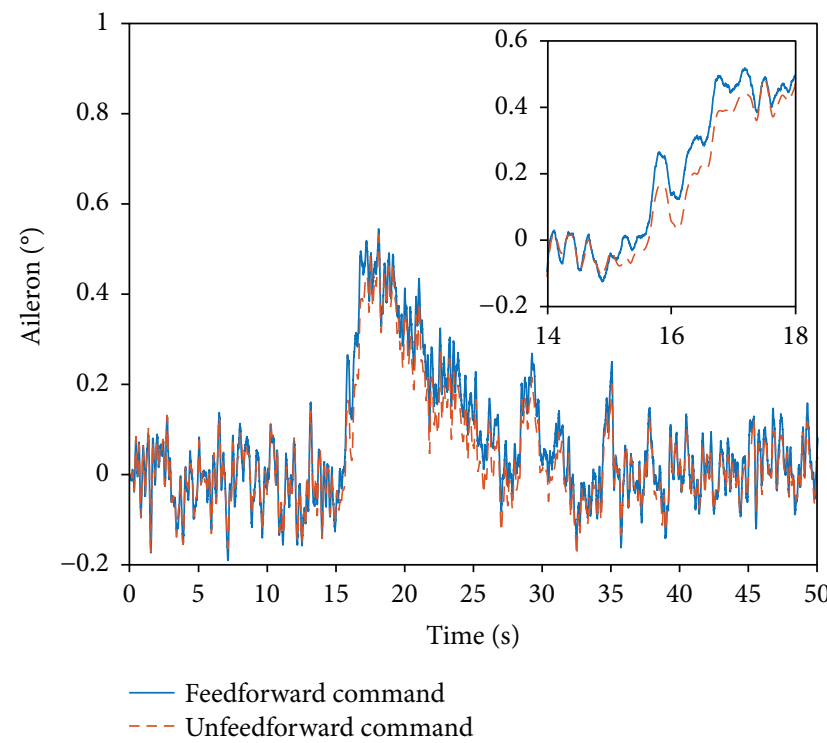

(a) The $+10 \mathrm{~m} / \mathrm{s}$ lateral gust

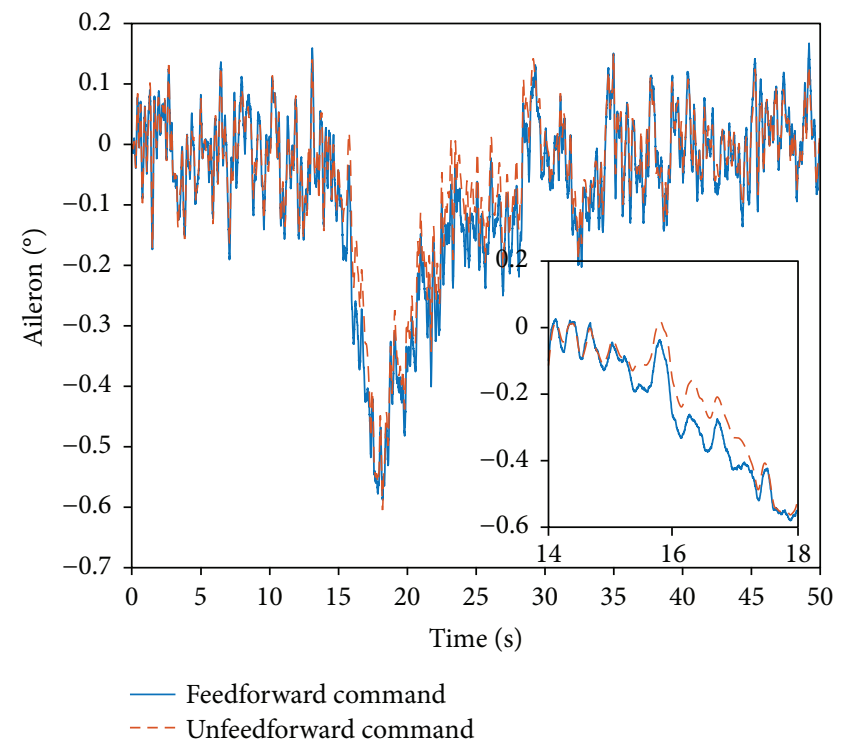

(b) The $-10 \mathrm{~m} / \mathrm{s}$ lateral gust

FIgURE 9: The control commands.

Figure 7 presents the control simulation results on the linear rolling controller. The red dashed line shows the simulation results on feedback control (unfeedforward); the blue solid line shows the simulation results on the composite feedforward-feedback control (feedforward). The additional feedforward channel has the capability to suppress external disturbance. The comparison of the lines in Figure 7 shows that the composite control architecture can almost entirely suppress the low-frequency external disturbance, which the feedback controller can barely do.

5.2. The 6-DOF Control Simulation Instance. To obtain reliable simulation results, the $6-\mathrm{DOF}$ control simulation is carried out. The real-time aerodynamic characteristics and pressure distribution are calculated with the AVL program package. The gusty environment is produced by combining the wing shear model, the Dryden wind turbulence model and the discrete wind gust model in the Simulink. A lateral gust is applied to the SUAV in 15 seconds.

Figures 8 and 9 show the 6-DOF control simulation results. Figures $8(\mathrm{a})$ and $8(\mathrm{~b})$ show the Euler rolling angle responses of the $10 \mathrm{~m} / \mathrm{s}$ and $-10 \mathrm{~m} / \mathrm{s}$ lateral gust, respectively. The red dashed line shows the simulation results on feedback control (unfeedforward); the blue solid line shows the simulation results on composite feedforward-feedback control (feedforward). The additional feedforward controller shows 
a large advantage for gust perturbation alleviation. Figure 9 shows the composite command and the conventional feedback command for rolling motion control. The composite controller offers the phase-advance commands.

However, beginning with the feedforward responses of the lateral gust (the blue solid line in 16 seconds), the SUAV seems to roll in the opposite direction. Obviously, the gustinduced rolling moment is overestimated maybe because of the aileron deflection and the tail.

\section{Conclusions}

Because a SUAV is more susceptible to rolling motion than to pitch and yaw motions, we propose a gust perturbation alleviation controller to enhance the rolling motion stability based on real-time flow pressure information. Two pairs of pressure sensors are embedded on the upper and lower surfaces of a SUAV's main wing. Each pair of sensors is positioned at the upper and lower surfaces at the same location. The position is elaborately selected at the $1 / 4$ wingspan from the wing tip and at the $25 \%$ chord length from the leading edge. The gust-induced rolling moment is estimated based on information obtained with pressure sensors. A feedforward controller for disturbance compensation is added to the traditional feedback controller. The advantage of using pressure sensors over inertial sensors is that they include the phase-advance information on aircraft dynamics. The comparison of composite feedforward control simulation results with feedback control simulation results shows that the proposed pressure sensor-based control method can mitigate low-frequency external perturbation.

The simulation results presented in the paper rely heavily on presumptions about sensor implementation. All aerodynamic data are obtained with the vortex-lattice computational method. More accurate estimation methods and more reliable flight tests should be further investigated in the future.

\section{Conflicts of Interest}

The authors declare that there is no conflict of interest regarding the publication of this article.

\section{Acknowledgments}

This work is supported by NSAF (no. U1630127).

\section{References}

[1] T. J. Mueller, "Overview of micro-air-vehicle development," in Introduction to the Design of Fixed-Wing Micro Air Vehicles Including Three Cases Studies, pp. 1-38, American Institute of Aeronautics and Astronautics, Reston, VA, USA, 2006.

[2] T. Tjahjowidodo and S. Lee, "Tendon-sheath mechanisms in flexible membrane wing mini-UAVs: control and performance," International Journal of Aerospace Engineering, vol. 2017, Article ID 8181743, 18 pages, 2017.

[3] K. Xue, C. Wang, Z. Li, and H. Chen, "Online adaptive error compensation SVM-based sliding mode control of an unmanned aerial vehicle," International Journal of Aerospace Engineering, vol. 2016, Article ID 8407491, 14 pages, 2016.

[4] E. Kayacan, M. A. Khanesar, J. Rubio-Hervas, and M. Reyhanoglu, "Learning control of fixed-wing unmanned aerial vehicles using fuzzy neural networks," International Journal of Aerospace Engineering, vol. 2017, Article ID 5402809, 12 pages, 2017.

[5] A. Mohamed, K. Massey, S. Watkins, and R. Clothier, "The attitude control of fixed-wing MAVS in turbulent environments," Progress in Aerospace Science, vol. 66, pp. 37-48, 2014.

[6] H. Shen, Y. Xu, and B. T. Dickinson, "Fault tolerant attitude control for small unmanned aircraft systems equipped with an airflow sensor array," Bioinspiration \& Biomimetics, vol. 9, no. 4, article 046015, 2014.

[7] D. Yeo, E. M. Atkins, L. P. Bernal, and W. Shyy, "Fixed-wing unmanned aircraft in-flight pitch and yaw control moment sensing," Journal of Aircraft, vol. 52, no. 2, pp. 403-420, 2015.

[8] S. A. Araujo-Estrada, F. Salama, C. M. Greatwood, K. T. Wood, T. S. Richardson, and S. P. Windsor, "Bio-inspired distributed strain and airflow sensing for small unmanned air vehicle flight control," in AIAA Guidance, Navigation, and Control Conference, pp. 1-19, Grapevine, TX, USA, 2017.

[9] R. Thompson, J. Evers, and K. Stewart, "Attitude control augmentation using wing load sensing - a biologically motivated strategy," in AIAA Atmospheric Flight Mechanics Conference, pp. 1-10, Toronto, ON, Canada, 2010.

[10] L. Castano, S. Airoldi, T. McKenna, and J. Humbert, "Gust rejection using force adaptive feedback for roll," in 14th AIAA Aviation Technology, Integration, and Operations Conference, pp. 1-15, Atlanta, GA, USA, 2014.

[11] G. M. Gremillion, L. Castano, and J. Humbert, "Disturbance rejection with distributed acceleration and strain sensing," in AIAA Guidance, Navigation, and Control Conference, pp. 1-17, Kissimmee, FL, USA, 2015.

[12] G. J. Rabadan, N. P. Schmitt, T. Pistner, and W. Rehm, “Airborne Lidar for automatic feedforward control of turbulent in-flight phenomena," Journal of Aircraft, vol. 47, no. 2, pp. 392-403, 2010.

[13] J. Zeng, B. Moulin, R. D. Callafon, and M. Brenner, “Adaptive feedforward control for gust load alleviation," Journal of Guidance, Control, and Dynamics, vol. 33, no. 3, pp. 862-872, 2010.

[14] J. Vogel and A. Kelkar, "Aircraft control augmentation and health monitoring using flush air data system feedback," in 26th AIAA Applied Aerodynamics Conference, pp. 1-15, Honolulu, HI, USA, 2008.

[15] M. Marino, S. Watkins, R. Sabatini, and A. Gardi, "Unsteady pressure measurements on a MAV wing for the design of a turbulence mitigation system," in 2014 IEEE Metrology for Aerospace (MetroAeroSpace), pp. 138-143, Benevento, Italy, May 2014.

[16] A. Mohamed, S. Watkins, A. Fisher, M. Marino, K. Massey, and R. Clothier, "Bioinspired wing-surface pressure sensing for attitude control of micro air vehicles," Journal of Aircraft, vol. 52, no. 3, pp. 827-838, 2015.

[17] M. Thompson, S. Watkins, C. White, and J. Holmes, "Spanwise wind fluctuations in open terrain as applicable to small flying craft," The Aeronautical Journal, vol. 115, no. 1173, pp. 693-701, 2011.

[18] M. Drela and H. Youngren, "Athena Vortex Lattice (AVL) software package, ver 3.35,” May 2018, http://web.mit.edu/ drela/Public/web/avl/. 


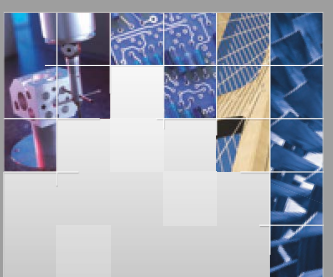

\section{Enfincering}
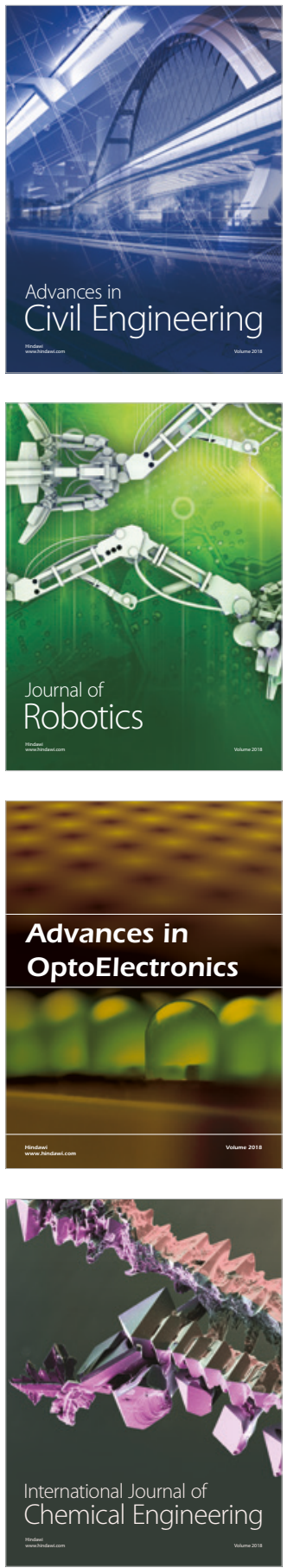

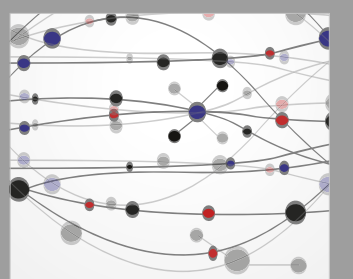

\section{Rotating \\ Machinery}

The Scientific World Journal

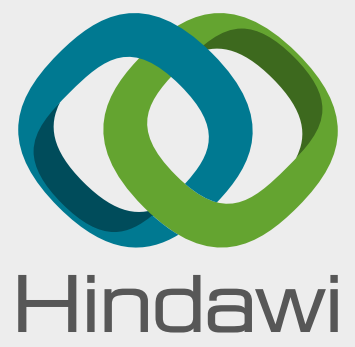

Submit your manuscripts at

www.hindawi.com
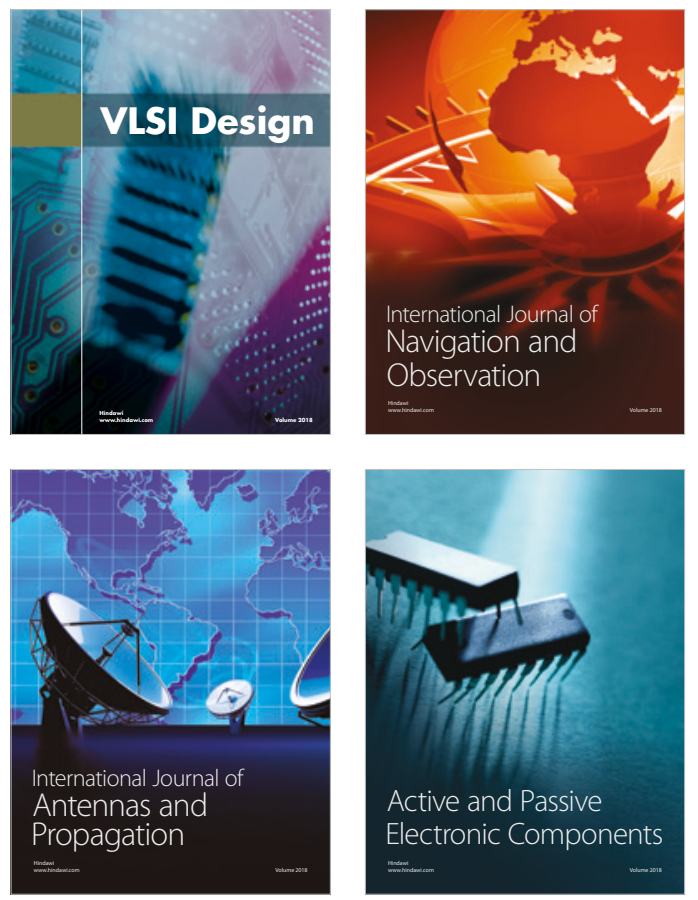
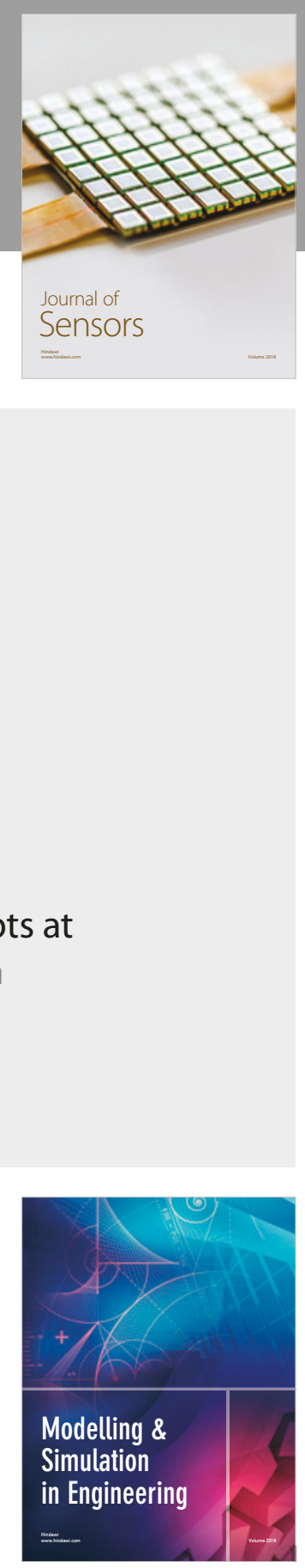

\section{Advances \\ Multimedia}
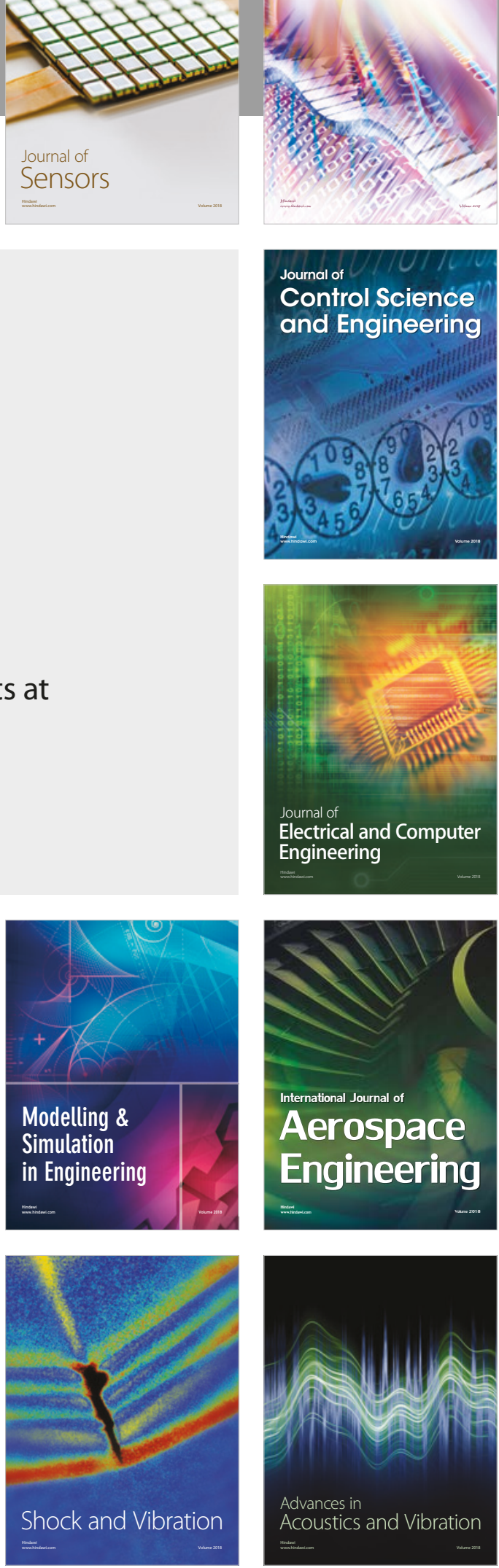